\title{
Therapeutic potential of capillary morphogenesis gene 2 extracellular vWA domain in tumour-related angiogenesis
}

\author{
LIN YE, PING-HUI SUN, ANDREW J. SANDERS, TRACEY A. MARTIN, \\ JANE LANE, MALCOLM D. MASON and WEN G. JIANG
}

\begin{abstract}
Metastasis and Angiogenesis Research Group, Cardiff University-Peking University Cancer Institute, Institute of Cancer and Genetics, Cardiff University School of Medicine, Cardiff, UK
\end{abstract}

Received March 12, 2014; Accepted May 14, 2014

DOI: $10.3892 /$ ijo.2014.2533

\begin{abstract}
Capillary morphogenesis gene 2 (CMG2) is a receptor of anthrax toxin and plays an important role in angiogenesis. It has been shown to be involved in the cell adhesion and motility of various cell types, including epithelia and endothelia. The present study aimed to examine the therapeutic potential of targeting CMG2 to prevent tumour-related new vasculature. The full-length coding sequence of the human CMG2 gene and different fragments of the CMG2 vWA domain were amplified and constructed into a mammalian expression plasmid vector. The effect of CMG2 and its vWA domain on endothelial cells and angiogenesis was assessed using relevant in vitro, ex vivo and in vivo models. The overexpression of $\mathrm{CMG} 2$ enhanced the adhesion of endothelial cells to extracellular matrix, but was negatively associated with cell migration. Overexpression of CMG2 and the vWA domain fragments inhibited the tubule formation and migration of endothelial cells. Small peptides based on the amino acid sequence of the CMG2 vWA domain fragments potently inhibited in vitro tubule formation and ex vivo angiogenesis. One of the polypeptides, LG20, showed an inhibitory effect on in vivo tumour growth of cancer cells which were co-inoculated with the vascular endothelial cells. CMG2 is a potential target for treating tumour-related angiogenesis. The polypeptides based on the CMG2 vWA domain can potently inhibit in vitro and ex vivo angiogenesis, which may contribute to the inhibitory effect on in vivo tumour growth. Further investigations are required to shed light on the machinery and may provide a novel therapeutic approach for inhibition of angiogenesis in cancer management.
\end{abstract}

Correspondence to: $\mathrm{Dr}$ Lin Ye, Metastasis and Angiogenesis Research Group, GF46 Henry Wellcome Building, Cardiff UniversityPeking University Cancer Institute, Institute of Cancer and Genetics, Cardiff University School of Medicine, Cardiff, CF14 4XN, UK E-mail: yel@cf.ac.uk

Key words: capillary morphogenesis gene 2, cancer, angiogenesis and therapy

\section{Introduction}

Capillary morphogenesis gene $2(C M G 2)$ also known as anthrax toxin receptor 2 (ANTXR2) has been identified as a gene upregulated in endothelial cells during tubule formation (1). CMG2 and tumour endothelial marker-8 (TEM-8) are receptors of anthrax toxin mediating the internalisation of the toxin $(2,3)$. CMG2 and TEM-8 are type I transmembrane proteins possessing an extracellular integrin-like I domain and are members of the von Willebrand factor A (vWA) domain-containing protein family $(4,5)$. The proteins share $40 \%$ overall amino acid identity, with $60 \%$ identity within their I domains, including a conserved metal ion dependent adhesion site (MIDAS) motif. The vWA/I domain with the MIDAS region, existing in different isoforms, allows the binding to protective antigen (PA) subunit of anthrax toxin which mediates the internalisation of the toxin. In addition to the binding with PA, the extracellular domain also interacts with collagen IV, laminin and fibronectin (1). The CMG2 gene is located on chromosome 4q, and encodes a 489-amino acid (aa) protein. The full-length protein has a putative signal peptide, extracellular, transmembrane and cytoplasmic domains (2). Apart from $\mathrm{CMG}_{2}{ }^{489}$, there are another three different natural variants encoded by alternatively spliced mRNA transcripts. CMG2 $2^{488}$ has 12 different amino acids at the cytoplasmic tail of the protein compared with $\mathrm{CMG} 2^{489} . \mathrm{CMG}^{386}$ lacks amino acids $213-233$ of the full length protein. $\mathrm{CMG}^{322}$ has been predicted to be a secreted isoform due to the lack of the transmembrane domain (2). CMG2 is more widely expressed in normal tissues except for brain and thymus (2), compared with TEM8 which is more selectively overexpressed during tumour angiogenesis. The finding of TEM8 as a specific tumour endothelial cell marker has led researchers, including ourselves, to investigate their role in the angiogenesis of malignancies. Since 2001, the host laboratory has started an investigation of its role in tumour-related angiogenesis and the interaction with other tumour-related cytokines and growth factors. The relationships of TEM-8 expression with clinical outcomes and the corresponding prognostic value have been evaluated in colorectal cancer and breast cancer (6-9). Elevated TEM8 expression in human colon cancer is associated with lymphatic metastasis and disease progression (7). Although TEM-8 might not be a marker specifically expressed in tumour-related endo- 
thelia, as it had been previously claimed to be, it is still a useful marker for identifying tumour associated micro-vessels and its elevated levels are associated with disease progression of breast cancer (9).

Mutations of CMG2 gene have been identified in juvenile hyaline fibromatosis (JHF) and infantile systemic hyalinosis (ISH) which are autosomal recessive syndromes characterized by multiple, recurring subcutaneous tumours, gingival hypertrophy, joint contractures, osteolysis and osteoporosis (10-14). The mutations result in low CMG2 mRNA and protein production due to the different cytosolic tails of protein products which can direct the proteins to endoplasmic reticulum (ER) associated degradation pathway (15). On the other hand, the mutation or variants of CMG2 may also affect sensitivity to anthrax toxin and lead to a reduced susceptibility to infection of Bacillus anthracis (16). Mutations like single nucleotide polymorphisms (SNPs) of CMG2 have also been associated with ankylosing spondylitis (AS) risk in Caucasians (17), but this was not evident in a cohort of 309 AS patients in Chinese Han population $(18,19)$. CMG2 knockout female mice were unable to produce any offspring due to a defect in parturition. This defect was caused by a diffuse deposition of collagen within the myometrium. The deletion of CMG2 did not affect normal mouse embryonic development (20). A recent study showed that CMG2 and TEM-8 were able to regulate the extracellular matrix via a regulation of MT1-MMP and MMP2 (21). This evidence suggests that $\mathrm{CMG} 2$ functions as a collagen receptor to maintain collagen homeostasis.

CMG2 has been shown to be able to regulate the proliferation and tubule formation of endothelial cells, but not the migration. This may have certain implication in tumour-related angiogenesis (22). In the present study we examined the impact of CMG2 on the angiogenic capacity of vascular endothelial cells and the possibility of targeting CMG2 vWA domain to interfere with tumour-related angiogenesis.

\section{Materials and methods}

Materials and cell lines. HECV cells purchased from Interlab (Milan, Italy) were maintained in Dulbecco's modified Eagle's medium (DMEM) (Sigma-Aldrich, Poole, Dorset, UK) supplemented with benzylpenicillin, amphotericin B, streptomysin and $10 \%$ foetal bovine serum (Sigma-Aldrich). The cells were incubated at $37^{\circ} \mathrm{C}, 5 \% \mathrm{CO}_{2}$ and $95 \%$ humidity. Matrigel was purchased from Collaborative Research Products (Bedford, MA, USA). Cell lines and human tissue cDNA libraries were prepared and stored in the host laboratory. Polyclonal goat anti-human-CMG2 was obtained from R\&D Systems (Minneapolis, MN, USA). Small polypeptides (as shown in Table I) were customised products synthesized by GeneCust Europe-Labbx (Luxembourg).

$R N A$ extraction and RT-PCR. RNA was extracted using total RNA isolation (TRI) reagent and following the protocol provided (Sigma-Aldrich). RNA was subsequently quantified using a spectrophotometer (WPA UV 1101, Biotech Photometer, Cambridge, UK), at $500 \mathrm{ng}$ of total RNA before being converted to cDNA using an iScript cDNA synthesis kit (Bio-Rad Laboratories, Hemel Hempstead, UK). The quality of cDNA was verified using GAPDH primers (sense 5'-CAGGAGGTT GAAGGACTAAA and antisense 5'-GGGATCAGTTTTCTT TGTCA). Conventional PCR was performed with specific primers for CMG2 (sense 5'-CAAAATCAGTAAAGGCT TGG, and antisense 5'-CAAAGGTTCTTCTTCCTCCT). The conditions for the amplification were: $94^{\circ} \mathrm{C}$ for $5 \mathrm{~min}$, followed by 35 cycles of $94^{\circ} \mathrm{C}$ for $30 \mathrm{sec}, 55^{\circ} \mathrm{C}$ for $30 \mathrm{sec}$ and $72^{\circ} \mathrm{C}$ for $1 \mathrm{~min}$, and the final extension for $7 \mathrm{~min}$ at $72^{\circ} \mathrm{C}$. The products were visualized on a $1.5 \%$ agarose gel after staining with ethidium bromide.

Construction of plasmid vectors carrying CMG2 sequences coding the full-length protein or vWA domain fragments. Full length of human CMG2 coding sequence was amplified from a cDNA library of human ovarian tissues stored at the host lab using PCR. The PCR products were purified and cloned into $\mathrm{pEF} / \mathrm{His}$ TOPO TA plasmid vector (Invitrogen, Inc., Paisley, $\mathrm{UK})$. Following transformation into $E$. coli and analysis of colonies, colonies carrying the correct inserts were amplified for extraction of the constructed plasmid vectors. The constructed CMG2 expression vectors were verified by sequencing before being used for the following experiments. The purified PCR products were also used to amplify different fragments of the CMG2 vWA domain which were then cloned into the same vector. The primer sequences are listed in Table II.

Transfection of the constructed vectors into human vascular endothelial cells. The constructed plasmid vectors carrying either full-length CMG2 or fragments of the vWA domain were used to transfect the HECV cells by way of electroporation. The empty plasmid vectors were transfected into HECV cells and used as a control for the following study. Following the transfection, the cells were selected using blasticidin $(5 \mu \mathrm{g} / \mathrm{ml})$. The cells were then cultured in DMEM with blasticidin at a lower concentration $(0.5 \mu \mathrm{g} / \mathrm{ml})$ to maintain the expression level.

Growth assay. A standard procedure was used as previously described $(23,24)$. Cells were plated into a 96-well plate (2,500 cells/well). Cell growth was assessed after 1, 3 and 5 days. Crystal violet was used to stain cells, and absorbance was determined at a wavelength of $540 \mathrm{~nm}$ using a spectrophotometer (BioTek, Elx800, UK).

Adhesion assay. This standard procedure was previously described (25). Cells $(40,000)$ were added in each well of a 96-well plate, previously coated with the Matrigel ( $5 \mu \mathrm{g} /$ well). After $40 \mathrm{~min}$ of incubation, non-adherent cells were washed off using BSS buffer. The number of adherent cells was then counted after fixation and staining.

In vitro motility assay using Cytodex-2 beads. We followed a protocol previously described $(26,27)$. Approximate $1 \times 10^{6}$ cells were incubated with $100 \mu \mathrm{l}$ of Cytodex beads in $10 \mathrm{ml}$ DMEM. After an overnight incubation, the beads were washed twice in $5 \mathrm{ml}$ DMEM to remove dead cells, and then resuspended in $800 \mu \mathrm{l}$ DMEM. A total of $100 \mu \mathrm{l}$ of beads/ cells was transferred into each well of a 24-well plate in triplicate. After incubation for $4 \mathrm{~h}$, the medium was aspirated and cells were fixed with $4 \%$ formalin for $5 \mathrm{~min}$. They were then stained with $0.5 \%$ crystal violet $(0.5 \%$ weight/volume in 
Table I. Amino acid sequence of the small peptides.

\begin{tabular}{ll}
\hline Name & \\
\hline LG20 & Lequence \\
LG64 & LDGLVPSYAEKEAKISRSLG \\
M1 & GLVPSYAEKEAKISRSLG \\
M2 & LVPSYAEKEAKISRSLG \\
M3 & LDGLVPSYAEKEAKISRS \\
M4 & LDGLVPSYAEKEAKIS \\
M5 & LVPSYAEKEAKISLG \\
M7 & VPSYAEKEAKISRSLG \\
M8 & PSYAEKEAKISRSLG \\
M9 & VPSYAEKEAKISR \\
M10 & SYAEKEAKISRSLG \\
\hline
\end{tabular}

Table II. Primer sequences for PCR.

\begin{tabular}{lll}
\hline & \multicolumn{1}{c}{ Forward } & Reverse \\
\hline CMG2 full length & ATGGTGGCGGAGCGGTCCCCGGCCCG & AGCAGTTAGCTCTTTCTCAATA \\
CMG2B1 & ATGGTGGCGGAGCGGTCCCCGGCCCG & TCTTCTGCAGGAGGGCTGCTCCT \\
CMG2B2 & ATGGTGGCGGAGCGGTCCCCGGCCCG & CTTGCCATCTGTCAGAGCAATTAT \\
CMG2B3 & ATGGTGGCGGAGCGGTCCCCGGCCCG & TAGTATAGAATTAATTATTCCTTTAAG \\
CMG2B4 & GCCTTTGATCTCTACTTCGT & TAGTATAGAATTAATTATTCCT \\
CMG2B5 & GCCTTTGATCTCTACTTCGT & CTTGCCATCTGTCAGAGC \\
CMG2B6 & GCCTTTGATCTCTACTTCGT & CCCAAGTGACCTGGATATCTT \\
CMG2B7 & TTGGACGGTCTGGTGCCAT & TAGTATAGAATTAATTATTCC
\end{tabular}

distilled water) for $5 \mathrm{~min}$. The cells were washed and allowed to dry before counting.

Electric cell-substrate impedance sensing (ECIS). An ECIS instrument of 9600 model (Applied Biophysics Inc., NJ, USA) was used for migration assay in the study, as previous reported (28). 96W1E arrays were used in this study. HECV cells were seeded at 40,000 cells per well in $200 \mu$ l DMEM medium. The resistance at $30 \mathrm{kHZ}$ was recorded for $10 \mathrm{~h}$ after an electrical wounding, and data were analysed using ECIS-9600 software package.

Tubule formation. The processes used were modified from previously published methods (29,30). Briefly, 96-well plates were coated with $100 \mu \mathrm{l} /$ well of Matrigel (diluted in a 1 to 1 ratio with serum free medium) and incubated for $30 \mathrm{~min}$ to allow the gel to set. HECV $\left(4 \times 10^{4}\right.$ cells per well) were seeded onto the Matrigel layer. The cells were treated with the small peptides or medium alone for 4-6 h to allow tubules to form.

Aorta ring assay. In this assay, angiogenic vessels grow from a segment of the aorta, which was modified from previously described methods $(31,32)$. Briefly, mouse thoracic aorta was dissected, the fat layer and adventitia were removed, and rings approximately $1 \mathrm{~mm}$ in length were prepared. Individual rings were embedded in Matrigel $(2.5 \mathrm{mg} / \mathrm{ml})$, cast inside individual wells of a 96-well plate. Small peptides were directly added into the medium for culturing the aorta rings. Each group had three replicates and two independent experiments were performed to assess the effect of the small peptides. All aorta rings were cultured in DMEM supplemented with $10 \%$ foetal bovine serum except for the negative control group which used serum-free DMEM. Sprouting was observed by inspection under a phase contrast microscope over a period of 6 days.

Mouse xenograft tumour model. Female athymic nude mice (4-8 weeks old; CD1; Charles River Laboratories) were subcutaneously (s.c.) injected with a mixture of cancer cells $\left(5 \times 10^{5}\right)$ and HECV cells $\left(5 \times 10^{5}\right)$ in Matrigel $(2.5 \mathrm{mg} / \mathrm{ml})$. Small peptides were given via intraperitoneal injection (i.p.). The mice were kept in sterilised, filtered cages in 12-h dark/12-h light standardized environmental conditions approved by Cardiff University Research Ethics Committee (UREC). Tumours were measured twice a week using digital callipers and calculated as tumour volume $=0.512 \mathrm{x}$ width ${ }^{2} \mathrm{x}$ length $\left(\mathrm{mm}^{3}\right)$. The protocol and procedure (project licence no. 30/2591) were approved by the Home Office, UK.

Statistical analysis. Two sample t-tests were performed using the SPSS statistical software (version 18, SPSS Inc. Chicago, IL, USA). Differences were considered to be statistically significant at $\mathrm{p}<0.05$. 


\section{Results}

The expression of CMG2 and TEM-8 in cell lines and human tissues. The expression of CMG2 was examined in human vascular endothelial cells (HECV), bladder cancer cell line (EJ-138), fibroblast cells (MRC-5), prostate cancer cell line (CAHPV-10) and breast cancer cell lines (ZR751, MCF-7 and MDA-MB-231) using RT-PCR (Fig. 1A). CMG2 transcripts are expressed in breast cancer cell lines, including ZR751, MCF-7 and MDA-MB-231, in which MCF-7 has a much lower level compared with the others. CMG-2 is not detectable in HECV cells, which do express TEM-8. Both CMG-2 and TEM- 8 are expressed by CAHPV-10 cells . This provided information to plan both in vitro and in vivo experimental models for further investigations of the role played by CMG2 in cancer and angiogenesis.

The expression of both CMG2 and TEM-8 was also examined in various human tissues (Fig. 1B). CMG2 was expressed in the two of the three ovarian tissues, and is highly expressed in colon and spleen tissues. It is also detectable in prostate tissue. Compared with CMG2, TEM- 8 is more ubiquitously expressed in the tissues we examined.

Overexpression of CMG2 in HECV cells and the effect on cell adhesion and migration. To examine the effect of CMG2 on functions of vascular endothelial cells, we transfected the HECV cells with the constructed plasmid vector carrying full-length of CMG2. An overexpression of CMG2 was confirmed in the transfected cells compared with the control cells (Fig. 2A). Following the verification, the effect on in vitro cell proliferation was determined using an in vitro cell growth assay. No obvious effect on cell growth was seen in the CMG2 overexpression cells (data not shown). The overexpression of CMG2 resulted in an enhanced cell-matrix adhesion of HECV cells (Fig. 2B). An opposite effect was seen in the cell motility which was determined using an in vitro beads assay (Fig. 2C).

Targeting extracellular $v W A$ domain of CMG2 to interfere with angiogenesis. vWA domain has been identified as a pivotal domain in regulation of cell functions, such as cellmatrix adhesion and cell motility. The vWA domain of TEM- 8 and CMG2 has been indicated as a key domain in control of adhesion and in vitro tubule formation of endothelial cells. Coding sequence of the vWA domain was divided into 6 fragments. A set of primers were designed to amplify 6 different products including these 6 fragments (Fig. 3A). Different fragmental sequences of the vWA domain were amplified from human ovarian tissue cDNA library, and cloned into plasmid vectors, respectively. The recombinant plasmid constructs and empty control plasmid vectors were then transfected into human vascular endothelial cells (HECV). The influence on cell function was examined using a series of in vitro functional assays, including cell growth, cell-matrix adhesion, motility and tubule formation.

The influence on tubule formation of HECV cells by overexpression of the full-length and different fragments of CMG2 was determined using the tubule formation assay. After the first stage of identification, the sequences of B6 and B7 and the common part of these two fragments were deduced

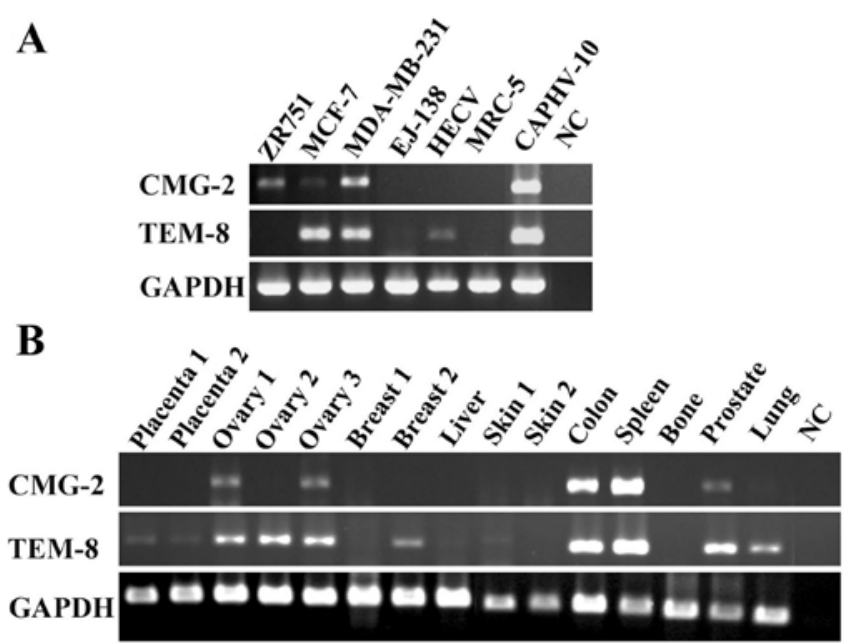

Figure 1. The expression of CMG2 and TEM-8 in cell lines and tissues. (A) The expression of CMG2 in cell lines. (B) CMG2 transcripts were determined in various tissues using RT-PCR.

to be potential candidates for further investigation. The overlapping part of CMG2-B6 and CMG2-B7 sequences was then amplified and cloned into the aforementioned plasmid vector. This fragment was named as CMG2 76. Its effect on tubule formation was assessed in the HECV cells which had this fragment overexpressed. Inhibition of tubule formation was seen in the cells overexpressed in the CMG2vWA domain fragments B3, B6, B7, B76 and the full-length CMG2 (Fig. 3B). Fragment B76 is an overlap sequence shared by the B6 and B7 fragments. The forced expression of these fragments and full-length of CMG2 suppressed the migration of endothelial cells in vitro (Fig. 3C). The B76 fragment exhibited a potent inhibitory effect on both migration and tubule formation of the endothelial cells.

Anti-angiogenic potential of small peptides based on the $v W A$ domain of $C M G 2$. Eleven polypeptides were then synthesised based on the amino acid sequence of CMG2 vWA domain fragment B76. The amino acid sequences of the polypeptides are provided in Table I. The influence on angiogenesis was then assessed using both in vitro tubule formation of HECV cells and ex vivo aorta assay. The cellular toxicity of these peptides was tested using in vitro cell growth assay, which indicated that these peptides are safe to human vascular endothelial cells over a range of concentration, from $60 \mathrm{pM}$ to $20 \mu \mathrm{M}$ (data not shown).

The effect on tubule formation of HECV cells was then assessed using the aforementioned method. In the experiments, LG20 and LL64 demonstrated better inhibitory effect on the in vitro tubule formation. The inhibition was seen in the endothelial cells exposed to a range of concentration from $640 \mathrm{pM}$ to $3 \mathrm{nM}$, the most obvious inhibition was seen at a concentration of $3 \mathrm{nM}$ (Fig. 4). The anti-angiogenic effect of these polypeptides was also assessed using the aorta ring assay, in which marked inhibition of angiogenesis was seen in the aorta rings exposed to LG20, M3 and M10 compared with the control and other polypeptides (Fig. 5). 

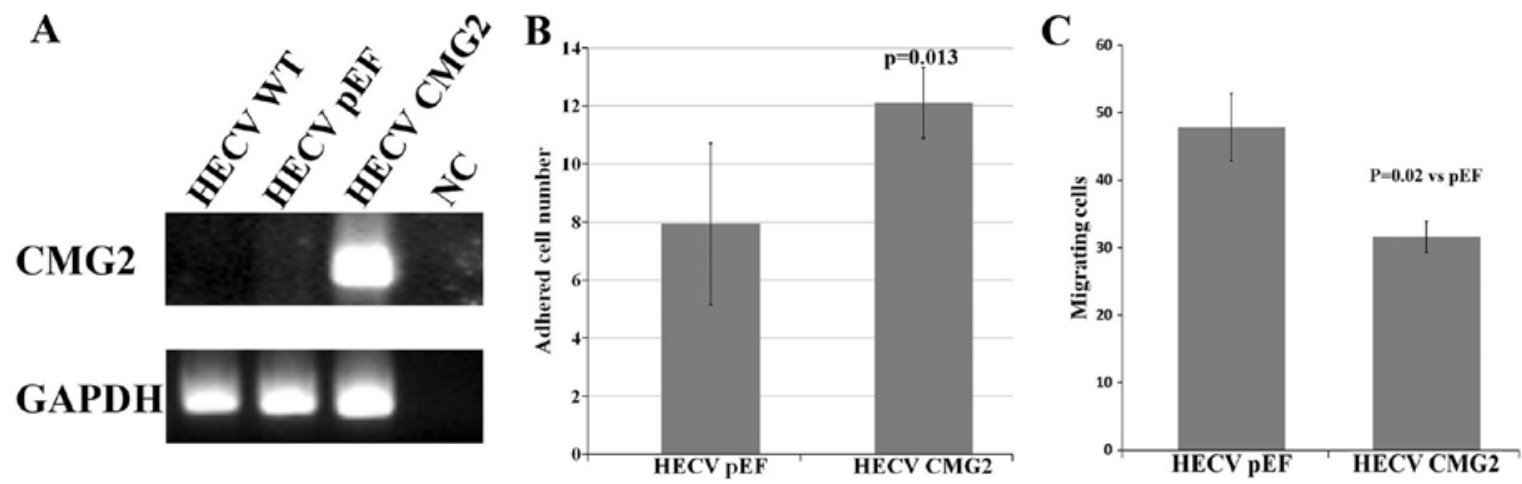

Figure 2. Effect on cellular functions of vascular endothelial cells (HECV) by CMG2 expression. (A) Forced overexpression of CMG2 was verified using RT-PCR. (B) The overexpression of CMG2 influenced the cell adhesion to extracellular matrix. (C) The effect of CMG2 on cell motility using an in vitro beads assay.

A

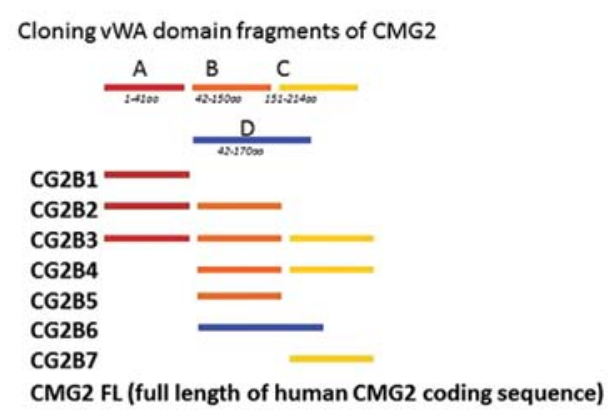

C

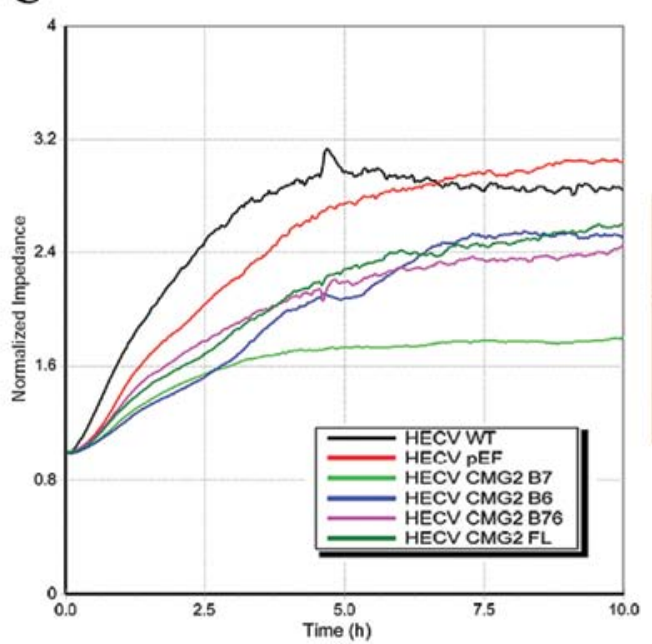

B

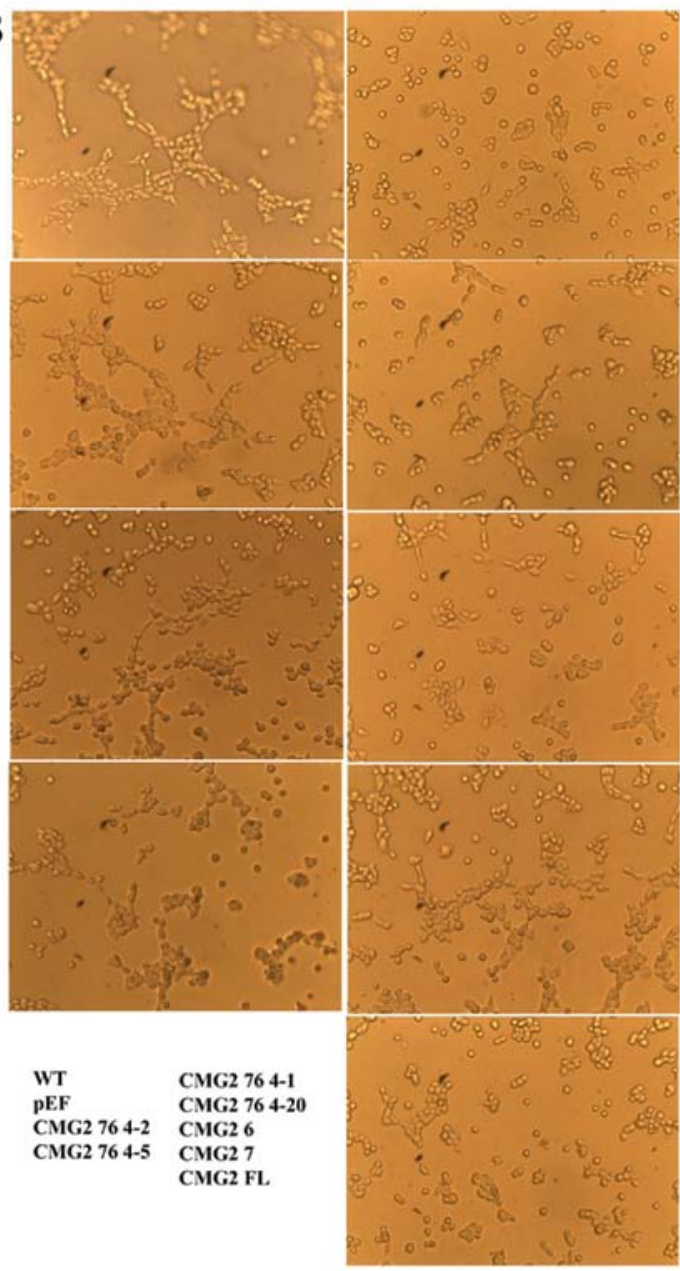

Figure 3. Different fragments of the CMG2 vWA domain and their effect on tubule formation of vascular endothelial cells. (A) A schematic diagram illustrates the cloning of different fragments of the CMG2 vWA domain. (B) Effect on the tubule formation. Fragments of CMG2 6, 7 and 76 a fragment overlap between CMG2 -6 and -7 fragments. (C) The effect of CMG2 vWA domain fragments on migration of vascular endothelial cells using ECIS.

The effect of LG20 on the in vivo tumour growth was then assessed. A prostate cancer cell line PC-3 was used to examine the effect on in vivo growth of prostate cancer cells. PC-3 cells and endothelia (HECV) were inoculated subcutaneously in nude mice. LG20 and control buffer (BSS) were injected via i.p. three times a week. A reduced tumour growth was seen in the mice receiving the LG20 treatment (Fig. 6A). After this study, we further assessed the therapeutic potential of LG20 in breast cancer. Breast cancer cells co-implanted with HECV cells and treated with LG20, did not affect the in vivo tumour growth as seen in the prostate cancer cells. However, when co-implanting the MCF-7 cells with HECV which overex- 


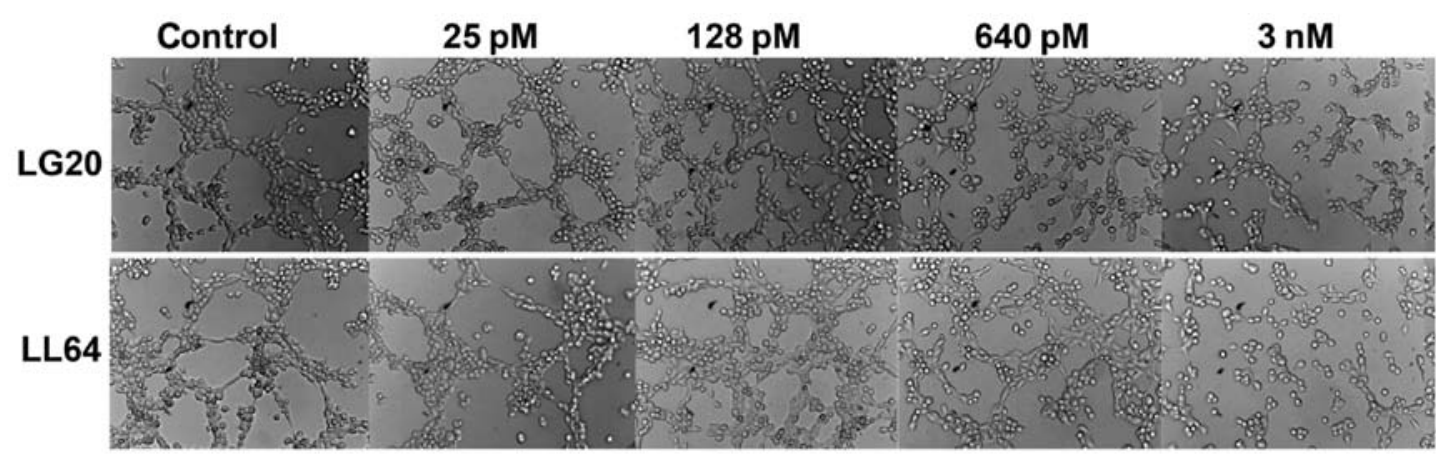

Figure 4. The effect on angiogenesis by the small peptides based on the amino sequence of CMG2 vWA domain using an ex vivo angiogenesis model.

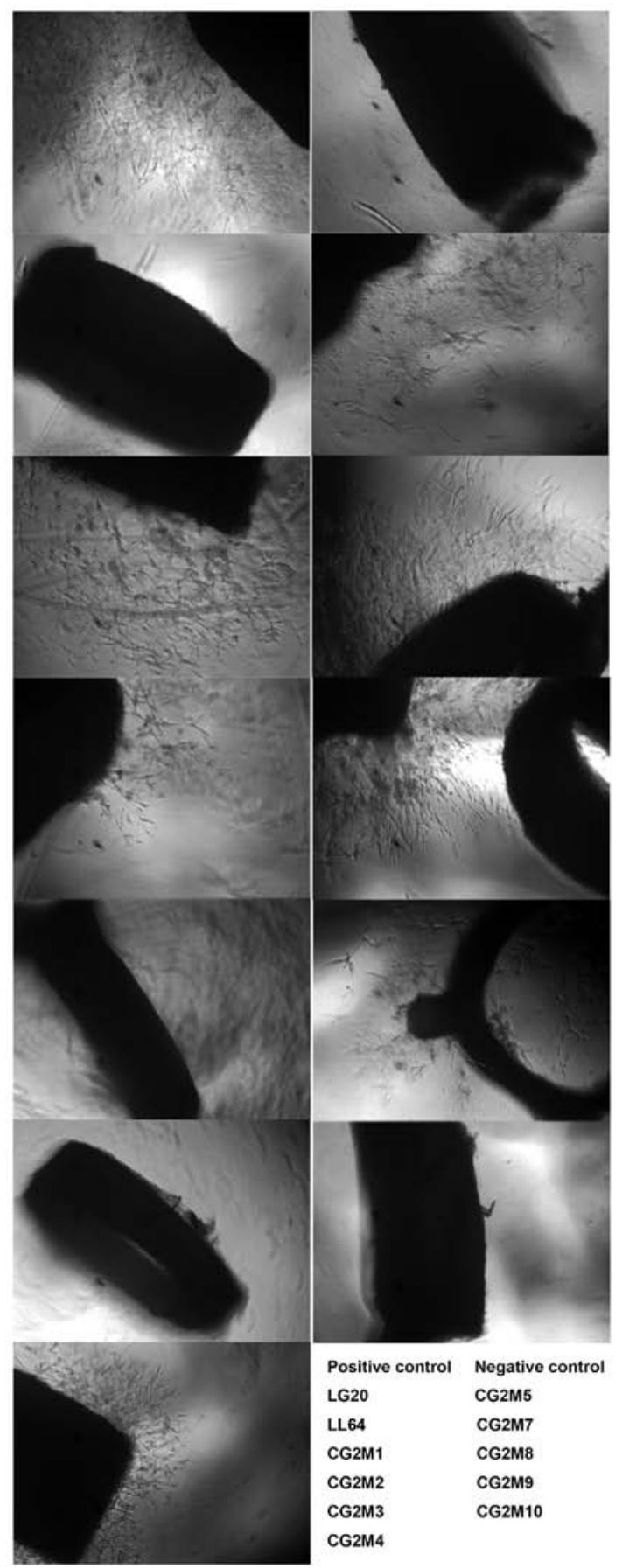

Figure 5. The effect on in vitro tubule formation by LG20 and LG64.
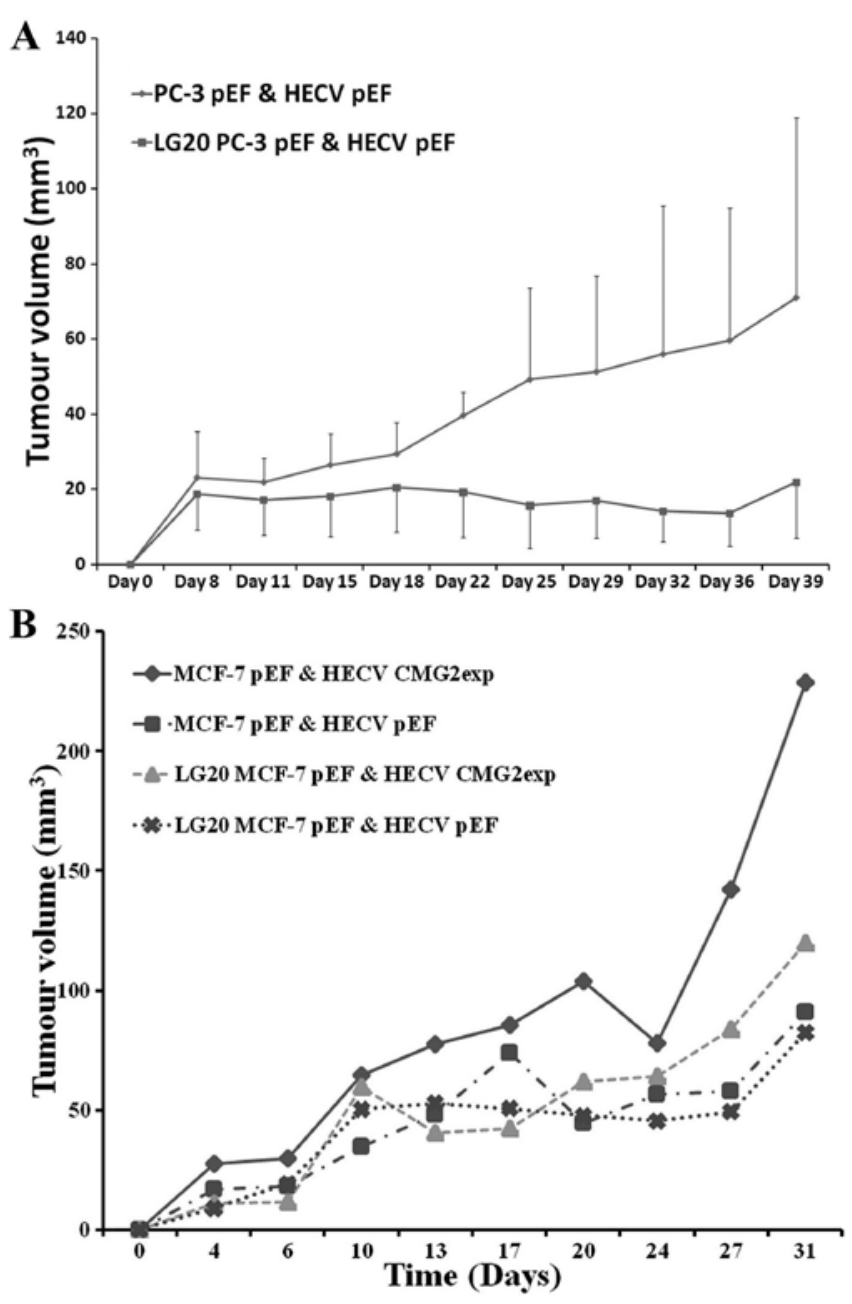

Figure 6. Effect on in vivo tumour growth by LG20. (A) LG20 suppressed tumour growth of PC-3 cells in vivo. (B) The inhibitory effect on HECV assisted tumour growth of MCF-7 cells by the intraperitoneal administration of LG20.

pressed CMG2, a remarkable increase of tumour growth was seen. This enhanced tumour growth was diminished by the LG20 treatment (Fig. 6B).

\section{Discussion}

CMG2 is a type I transmembrane protein possessing an extracellular integrin-like I domain, a member of the larger family 
of von Willebrand factor A domains $(4,5)$. The CMG2 and TEM- 8 proteins share $40 \%$ overall amino acid identity, with $60 \%$ identity within their I domains, including a perfectly conserved metal ion dependent adhesion site (MIDAS) motif. CMG2 is widely expressed in normal tissues (2), whereas TEM-8 was reported to be selectively overexpressed during tumour angiogenesis. In the current study, we examined the expression of CMG2 in cDNAs of cell lines and tissues. Three breast cancer cell lines (ZR751, MCF7 and MDA-MB231) and prostate cancer cell line (CAPHV10) are positive for the CMG2 expression. However, a vascular endothelial cell line (HECV) appears to be negative while TEM-8 appears to be positive in this endothelial cell line. CMG2 was also not detectable in the two placenta tissues which comprised of abundant new vasculature. Similarly, this absence was also evident in other tissues with abundant blood vessels such as the liver. However, highly positive expression of both CMG2 and TEM- 8 was seen in both spleen and colon tissues. This suggests CMG2 expression in the endothelial cells may have spatial-temporal variations during the angiogenic process and maturation of the blood vasculature. This requires further investigation into the expression of CMG2 and its functions in different tissues and endothelial cells at various phases according to the angiogenic process.

The discovery of TEM-8 as a specific tumour cell marker has raised great interest for researchers to develop anti-angiogenesis approaches. The potential and recent development of anti-angiogenesis therapy targeting TEM- 8 and CMG2, such as modified anthrax toxin and anti-TEM- 8 antibodies have been reviewed recently (33). CMG2 has been demonstrated as the major receptor of anthrax toxin mediating direct lethality (34). A binding of the CMG2 to domain 2 and 4 of PA may act as receptor-based molecular switch that controls anthrax toxin entry into cells (35). The binding to the domain 2 is weakened prior to pore-to-pore conversion whilst the binding to domain 4 remains the same during the conversion (35). Protective antigen (PA) is a non-pathogenic component of anthrax toxin and can also inhibit angiogenesis by interacting with CMG2 and TEM-8. For example, a form of modified PA with three mutated amino acids, PA-SSSR, can inhibit migration of endothelial cells and also angiogenesis in vivo (36). PA-SSSR can suppress VEGF and serum induced migration of microvascular endothelial cells (HMVEC) with no effect on their proliferation. PA-SSSR also inhibits angiogenesis in a corneal angiogenesis assay and growth of lung cancer cells. The modified PA, without the assistance of the lethal factor and oedema factor of anthrax toxin, suggests the binding of PA to its receptor CMG2 and TEM-8 can directly influence cellular functions via machinery yet to be investigated.

Research to develop a therapeutic approach targeting TEM- 8 has been underway in the host laboratory since 2002 . Hammer-head ribozyme transgenes targeting TEM- 8 could reduce the expression of TEM-8 in human vascular endothelial cells (HECV), and therefore prevent their in vitro tubule formation. On the other hand overexpression of different domains of TEM-8 has revealed that vWA domain played a key role in control of in vitro tubule formation, as well as the extracellular domain with transmembrane domain (37). This suggests a potential for targeting at the vWA domain of TEM-8.
CMG2 is upregulated in vascular endothelial cells during formation of new capillaries (1). A recent study showed that CMG2 inhibits the growth of vascular endothelial cells (HUVEC) leading to inhibition of angiogenic capacity of the endothelia with no obvious effect on cell migration. In the current study, the overexpression of CMG2 in HECV cells enhanced the adhesion to extracellular matrix, but was negatively associated with cell migration. Overexpression of certain fragments (extracellular domains) inhibited the tubule formation and migration of endothelial cells. Our data suggest a negative role played by CMG2 for the angiogenic capacity of HECV cells. The controversial findings indicate diverse functions possibly played by CMG2 at different stages of the angiogenic process. Differences in the endothelial cells examined in the studies may be a possible reason for the different findings, which may reflect the nature of endothelial cells from different collections.

CMG2 contains a signal peptide, an extracellular von Willebrand factor A (vWA) domain, a single-pass transmembrane region (TM) for plasma membrane anchoring, and a cytosolic tail that might be involved in cytoskeleton interaction and is subject to certain post-translational modifications $(38,39)$. CMG2 and TEM-8 share $60 \%$ sequence identity in their vWA domains, which contain a typical metal ion-dependent adhesion site (MIDAS) motif responsible for PA binding (39). The cytosolic tail of the TEM-8 can bind to filamentous actin which leads to a reduced association of the extracellular domain with PA (40).

The current study was carried out to examine the role played by CMG2 in tumour-related angiogenesis, and to develop an approach for anti-angiogenesis targeting CMG2. We examined the function of different fragments within $\mathrm{CMG} 2 \mathrm{vWA}$ domain by overexpressing these fragments in vascular endothelial cells. Experimental data showed an anti-angiogenesis effect by a specific fragment. A few polypeptides have been designed and synthesised based on this fragment. The effect against angiogenesis has been examined using in vitro and ex vivo angiogenesis models. Small peptides mimicking the amino acid sequence of the fragments potently inhibit the in vitro tubule formation and ex vivo angiogenesis. Tests of certain small peptides showed an inhibitory effect on in vivo tumour growth of cancer cells which we have examined. In addition to its crucial role in angiogenesis, our recent studies have also demonstrated direct impact of this molecule on cancer cells in prostate and breast cancer (unpublished data). Other recent studies have revealed an important role of the vWA domain for the functions of CMG2 protein, and great potential for antiangiogenesis by targeting this domain. For example, TEM-8 and CMG2 extracellular domain, particularly vWA domain based decoys can be used as anthrax toxin inhibitors (41).

In addition to the above approaches, natural molecules targeting CMG2 have been assessed for their anti-angiogenic potential. For example, PGG (1,2,3,4,6-penta-O-galloyl- $\beta$ $\mathrm{D}$-glucopyranose) is a gallotannin produced by a variety of medicinal plants and has antitumour effects. It has been recently shown as a CMG2 inhibitor with anti-angiogenic activity using a high-throughput fluorescence resonance energy transfer (FRET) based screening assay (42). PGG can inhibit migration of human dermal microvascular endothelial cells which together with its other antitumour activities may contribute to the inhibition of in vivo tumour growth. The 
same research team has also identified some other inhibitors which may target CMG2 and can be used for anti-angiogenic therapy. These inhibitors include compounds from CR252M and CR1207B. The CR252M is an endophytic fungus Coccomyces proteae collected from a Costa Rican rainforest, and the CR1207B is Aurapex penicillata (43). The first inhibitor identified using the FRET was tannic acid which can interact with CMG2 and suppressed angiogenesis (44).

The present data have demonstrated great therapeutic potential of the CMG2 vWA fragment for tumour-related angiogenesis. However, further investigations are required to elucidate the mechanisms underlying their anticancer and anti-angiogenesis effects, and examine the safety of novel therapeutic reagents and improve their efficiency and specificity towards a real drug against tumour-associated angiogenesis.

In summary, CMG2 is a potential target of anti-angiogenic therapy. Small peptides based on the extracellular vWA domain of CMG2 can potently inhibit angiogenesis in vitro and ex vivo, which may contribute to its inhibitory effect on the in vivo tumour growth. Further investigations will shed light on the underlying mechanisms and help to fully establish the therapeutic potential of targeting CMG2 vWA domain to prevent tumour-related angiogenesis.

\section{Acknowledgements}

The authors thank Cancer Research Wales for the great support to this study.

\section{References}

1. Bell SE, Mavila A, Salazar R, et al: Differential gene expression during capillary morphogenesis in 3D collagen matrices: regulated expression of genes involved in basement membrane matrix assembly, cell cycle progression, cellular differentiation and G-protein signaling. J Cell Sci 114: 2755 2773, 2001.

2. Scobie HM, Rainey GJ, Bradley KA and Young JA: Human capillary morphogenesis protein 2 functions as an anthrax toxin receptor. Proc Natl Acad Sci USA 100: 5170-5174, 2003

3. Bradley KA, Mogridge J, Mourez M, Collier RJ and Young JA: Identification of the cellular receptor for anthrax toxin. Nature 414: 225-229, 2001.

4. Carson-Walter EB, Watkins DN, Nanda A, Vogelstein B, Kinzler KW and St Croix B: Cell surface tumor endothelial markers are conserved in mice and humans. Cancer Res 61: 6649-6655, 2001.

5. Emsley J, King SL, Bergelson JM and Liddington RC: Crystal structure of the I domain from integrin alpha2beta1. J Biol Chem 272: 28512-28517, 1997.

6. Davies G, Cunnick GH, Mansel RE, Mason MD and Jiang WG: Levels of expression of endothelial markers specific to tumourassociated endothelial cells and their correlation with prognosis in patients with breast cancer. Clin Exp Metastasis 21: 31-37, 2004.

7. Rmali KA, Watkins G, Harrison G, Parr C, Puntis MC and Jiang WG: Tumour endothelial marker 8 (TEM-8) in human colon cancer and its association with tumour progression. Eur $\mathbf{J}$ Surg Oncol 30: 948-953, 2004.

8. Rmali KA, Puntis MC and Jiang WG: Prognostic values of tumor endothelial markers in patients with colorectal cancer. World J Gastroenterol 11: 1283-1286, 2005.

9. Davies G, Rmali KA, Watkins G, Mansel RE, Mason MD and Jiang WG: Elevated levels of tumour endothelial marker-8 in human breast cancer and its clinical significance. Int $\mathrm{J}$ Oncol 29: 1311-1317, 2006.

10. Hanks S, Adams S, Douglas J, et al: Mutations in the gene encoding capillary morphogenesis protein 2 cause juvenile hyaline fibromatosis and infantile systemic hyalinosis. Am J Hum Genet 73: 791-800, 2003.
11. Dowling O, Difeo A, Ramirez MC, et al: Mutations in capillary morphogenesis gene-2 result in the allelic disorders juvenile hyaline fibromatosis and infantile systemic hyalinosis. Am J Hum Genet 73: 957-966, 2003.

12. Tumer L, Kasapkara C, Fong K, Serdaroglu A and McGrath JA: Hyaline fibromatosis syndrome resulting from a new homozygous missense mutation, p.Gly116Val, in ANTXR2. J Dermatol 40: 677-678, 2013.

13. Al Sinani S, Al Murshedy F and Abdwani R: Infantile systemic hyalinosis: a case report with a novel mutation. Oman Med J 28: 53-55, 2013.

14. Wang YY, Wen CQ, Wei Z and Jin X: A novel splice site mutation in ANTXR2 (CMG2) gene results in systemic hyalinosis. J Pediatr Hematol Oncol 33: e355-e357, 2011.

15. Yan SE, Lemmin T, Salvi S, et al: In-depth analysis of hyaline fibromatosis syndrome frameshift mutations at the same site reveal the necessity of personalized therapy. Hum Mutat 34: 1005-1017, 2013.

16. Martchenko M, Candille SI, Tang $\mathrm{H}$ and Cohen SN: Human genetic variation altering anthrax toxin sensitivity. Proc Natl Acad Sci USA 109: 2972-2977, 2012.

17. Reveille JD, Sims AM, Danoy P, et al: Genome-wide association study of ankylosing spondylitis identifies non-MHC susceptibility loci. Nat Genet 42: 123-127, 2010.

18. Guo C, Xia Y, Yang Q, Qiu R, Zhao H and Liu Q: Association of the ANTXR2 gene polymorphism and ankylosing spondylitis in Chinese Han. Scand J Rheumatol 41: 29-32, 2012.

19. Chen C, Zhang X and Wang Y: ANTXR2 and IL-1R2 polymorphisms are not associated with ankylosing spondylitis in Chinese Han population. Rheumatol Int 32: 15-19, 2012.

20. Peters DE, Zhang Y, Molinolo AA, et al: Capillary morphogenesis protein-2 is required for mouse parturition by maintaining uterine collagen homeostasis. Biochem Bioph Res Co 422: 393-397, 2012.

21. Reeves CV, Wang X, Charles-Horvath PC, et al: Anthrax toxin receptor 2 functions in ECM homeostasis of the murine reproductive tract and promotes MMP activity. PLoS One 7: e34862, 2012.

22. Reeves CV, Dufraine J, Young JA and Kitajewski J: Anthrax toxin receptor 2 is expressed in murine and tumor vasculature and functions in endothelial proliferation and morphogenesis. Oncogene 29: 789-801, 2010.

23. Bonnekoh B, Wevers A, Jugert F, Merk H and Mahrle G: Colorimetric growth assay for epidermal cell cultures by their crystal violet binding capacity. Arch Dermatol Res 281: 487-490, 1989.

24. Jiang WG, Davies G, Martin TA, et al: Targeting matrilysin and its impact on tumor growth in vivo: the potential implications in breast cancer therapy. Clin Cancer Res 11: 6012-6019, 2005.

25. Jiang WG, Hiscox S, Hallett MB, Scott C, Horrobin DF and Puntis MC: Inhibition of hepatocyte growth factor-induced motility and in vitro invasion of human colon cancer cells by gamma-linolenic acid. Br J Cancer 71: 744-752, 1995.

26. Rosen EM, Meromsky L, Setter E, Vinter DW and Goldberg ID: Smooth muscle-derived factor stimulates mobility of human tumor cells. Invasion Metastasis 10: 49-64, 1990.

27. Jiang WG, Hiscox S, Singhrao SK, Nakamura T, Puntis MC and Hallett MB: Inhibition of HGF/SF-induced membrane ruffling and cell motility by transient elevation of cytosolic free $\mathrm{Ca}^{2+}$. Exp Cell Res 220: 424-433, 1995.

28. Jiang WG, Martin TA, Lewis-Russell JM, Douglas-Jones A, Ye L and Mansel RE: Eplin-alpha expression in human breast cancer, the impact on cellular migration and clinical outcome. Mol Cancer 7: 71, 2008

29. Cai J, Jiang WG and Mansel RE: Inhibition of the expression of VE-cadherin/catenin complex by gamma linolenic acid in human vascular endothelial cells, and its impact on angiogenesis. Biochem Biophys Res Commun 258: 113-118, 1999.

30. Grant DS, Tashiro K, Segui-Real B, Yamada Y, Martin GR and Kleinman HK: Two different laminin domains mediate the differentiation of human endothelial cells into capillary-like structures in vitro. Cell 58: 933-943, 1989.

31. Cai J, Jiang WG and Mansel RE: Inhibition of angiogenic factor- and tumour-induced angiogenesis by gamma linolenic acid. Prostaglandins Leukot Essent Fatty Acids 60: 21-29, 1999.

32. Jiang WG and Harding KG: Enhancement of wound tissue expansion and angiogenesis by matrix-embedded fibroblast (dermagraft), a role of hepatocyte growth factor/scatter factor. Int J Mol Med 2: 203-210, 1998. 
33. Chaudhary A and St Croix B: Selective blockade of tumor angiogenesis. Cell Cycle 11: 2253-2259, 2012.

34. Liu S, Zhang Y, Hoover B and Leppla SH: The receptors that mediate the direct lethality of anthrax toxin. Toxins (Basel) 5: $1-8,2013$.

35. Pilpa RM, Bayrhuber M, Marlett JM, Riek R and Young JA: A receptor-based switch that regulates anthrax toxin pore formation. PLoS Pathog 7: e1002354, 2011.

36. Rogers MS, Christensen KA, Birsner AE, et al: Mutant anthrax toxin B moiety (protective antigen) inhibits angiogenesis and tumor growth. Cancer Res 67: 9980-9985, 2007.

37. Rmali KA, Puntis MC and Jiang WG: TEM- 8 and tubule formation in endothelial cells, its potential role of its vW/TM domains. Biochem Biophys Res Commun 334: 231-238, 2005.

38. Abrami L, Leppla SH and van der Goot FG: Receptor palmitoylation and ubiquitination regulate anthrax toxin endocytosis. J Cell Biol 172: 309-320, 2006.

39. Liu S, Leung HJ and Leppla SH: Characterization of the interaction between anthrax toxin and its cellular receptors. Cell Microbiol 9: 977-987, 2007.
40. Garlick KM, Batty S and Mogridge J: Binding of filamentous actin to anthrax toxin receptor 1 decreases its association with protective antigen. Biochemistry 51: 1249-1256, 2012.

41. Cai C, Che J, Xu L, et al: Tumor endothelium marker- 8 based decoys exhibit superiority over capillary morphogenesis protein-2 based decoys as anthrax toxin inhibitors. PLoS One 6: e20646, 2011.

42. Cryan LM, Bazinet L, Habeshian KA, et al: 1,2,3,4,6-PentaO-galloyl-beta-D-glucopyranose inhibits angiogenesis via inhibition of capillary morphogenesis gene 2. J Med Chem 56: 1940-1945, 2013

43. Cao S, Cryan L, Habeshian KA, et al: Phenolic compounds as antiangiogenic CMG2 inhibitors from Costa Rican endophytic fungi. Bioorg Med Chem Lett 22: 5885-5888, 2012.

44. Rogers MS, Cryan LM, Habeshian KA, et al: A FRET-based high throughput screening assay to identify inhibitors of anthrax protective antigen binding to capillary morphogenesis gene 2 protein. PLoS One 7: e39911, 2012. 